\title{
The Application of FastICA Combined with Related Function in Blind Signal Separation
}

\author{
Dengao Li, Junmin Zhao, Hongyan Liu, and Defeng Hao \\ College of Information Engineering, Taiyuan University of Technology, Taiyuan 030024, China \\ Correspondence should be addressed to Dengao Li; lidengaoty@126.com
}

Received 20 July 2013; Accepted 7 March 2014; Published 3 April 2014

Academic Editor: Vishal Bhatnagar

Copyright (C) 2014 Dengao Li et al. This is an open access article distributed under the Creative Commons Attribution License, which permits unrestricted use, distribution, and reproduction in any medium, provided the original work is properly cited.

\begin{abstract}
Blind source separation (BSS) has applications in the fields of data compression, feature recognition, speech, audio, and biosignal processing. Identification of ECG signal is one of the challenges in the biosignal processing. Proposed in this paper is a new method, which is the combination of related function relevance to estimated signal and negative entropy in fast independent component analysis (FastICA) as objective function, and the iterative formula is derived without any assumptions; then the independent components are found by maximizing the objective function. The improved algorithm shorthand for R-FastICA is applied to extract random mixed signals and ventricular late potential (VLP) signal from normal ECG signal; simultaneously the performance of RFastICA algorithm is compared with traditional FastICA through simulation. Experimental results show that R-FastICA algorithm outperforms traditional FastICA with higher similarity coefficient and separation precision.
\end{abstract}

\section{Introduction}

Blind source separation (BSS) [1] has been applied successfully to extract mixed signals in different fields of data compression [2], feature recognition [3], speech, audio, and biosignal processing $[4,5]$ as a statistical signal method. Literature [2] showed that the compression ratio was higher through the ICA method than principal component analysis (PCA). The accuracy ratio of feature recognition was $92.1 \%$ based on the complex valued Independent Component Analysis in literature [3]. Literature $[4,5]$ showed that FastICA was an efficient method in the extraction of speech, audio, and biosignal. Neither the source signals nor the structure of mixed matrix is known [6].

The detection and analysis of VLP generally appearing in the end of QRS wave and extending to ST segment with a series of high frequency and low-rising weak irregular electrical signal are a kind of effective means to predict unexplained asphyxia, sudden cardiac deaths, and so forth [7]. At present, the analysis methods of VLP commonly have had a time domain method, frequency domain method, spectrum scale measurement analysis method, and so forth [8]. Time domain method is not easy to improve detection rate of VLP; frequency domain analysis is limited by frequency resolution; spectrum scale measurement analysis can overcome some limitations in time domain and frequency domain analysis, but it is easily influenced by the selection of analysis time and positioning of QRS wave terminal in extracting judgmental standard parameters of VLP [9].

To overcome the abovementioned limitation and improve the detection accuracy, it is necessary to put forward a new detection technology. Independent component analysis (ICA) as a branch of BSS is widely applied to this problem in recent years [10-12]. Traditional FastICA algorithm has obtained several effects in extracting electromyographic and atrial fibrillation signal. VLP compared with normal ECG signal waveform has a relative independence, and ICA algorithm can accurately distinguish relatively independent component from the ECG; it can be used to identify VLP. In addition, many authors have conducted specific research to ICA algorithm. Literature [13] proposed an adaptive ICA algorithm based on artificial neural network, which reduced the complexity of obtaining the learning matrix and independent component; literature [14] designed a fast search algorithm directly based on kurtosis as a measure of nonGauss; literature [15] analyzed the principle and method of 


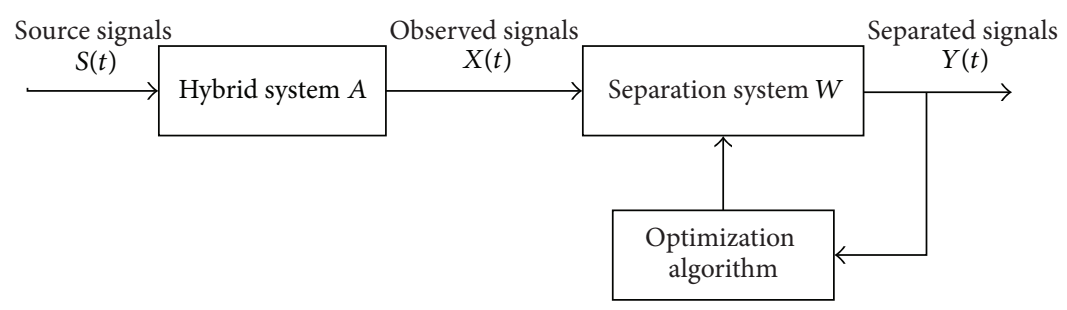

FIGURE 1: FastICA principle diagram.
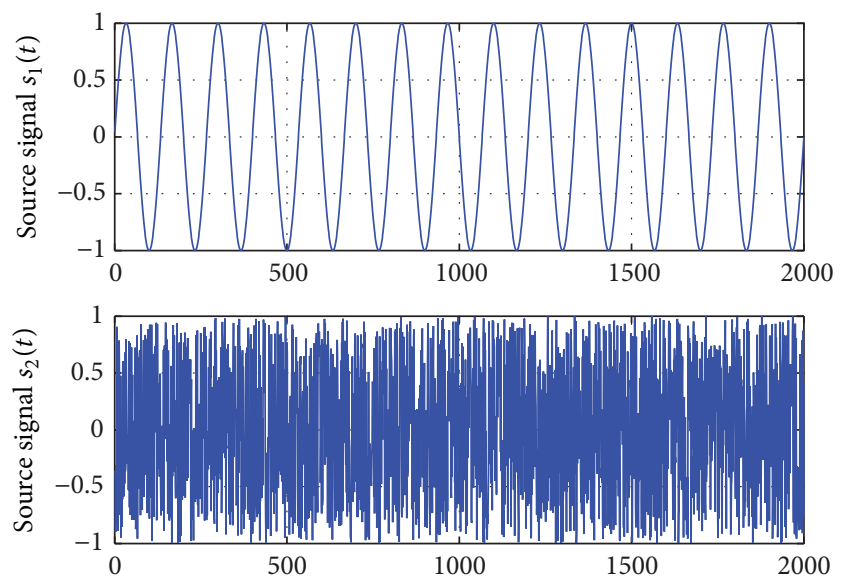

Figure 2: The source signals.
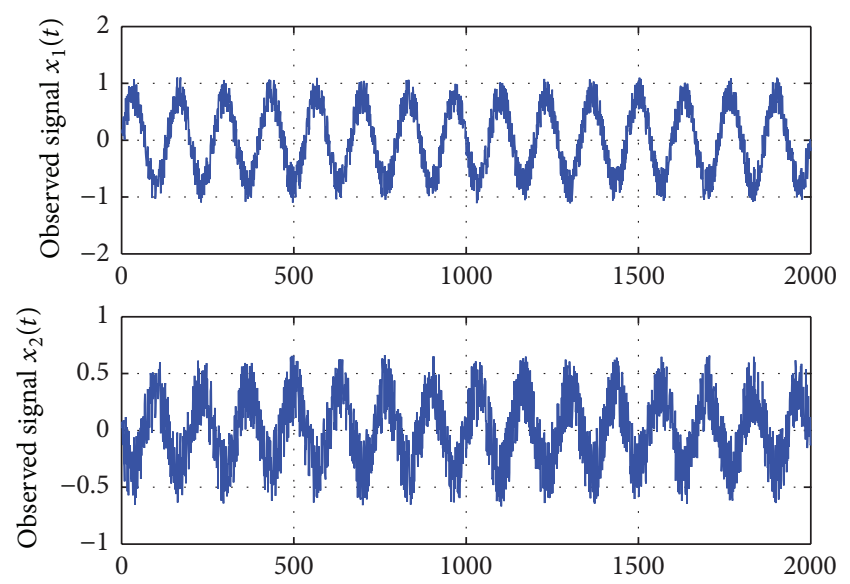

Figure 3: The mixed signals.

independent component in serial estimation ICA model; the concept of kernel ICA was proposed in literature [16], where data was analyzed through related analysis combined with mutual information theory; it had obtained better separation effect.

R-FastICA is proposed in this paper, which is the combination of related function and negative entropy as objective function, and the iterative formula is derived; then the independent components are found by maximizing the objective function. The extracted performance of R-FastICA algorithm is compared with traditional FastICA through simulation of random mixed signals and ECG signal with VLP. Experimental results show that R-FastICA algorithm outperforms traditional FastICA with higher similarity coefficient and separation precision.

\section{Theory}

ICA firstly proposed by Pierre Comon in 1994 is a method for finding the statistical independent components from multidimensional statistical data [17]. The mathematical model without noises can be expressed as follows:

$$
X(t)=A S(t),
$$

where $S(t)=\left[s_{1}(t), s_{2}(t), \ldots, s_{N}(t)\right]^{T}$ is a $N$ column vector of source signals and $X(t)=\left[x_{1}(t), x_{2}(t), \ldots, x_{\mathrm{M}}(t)\right]^{T}$ is $M$ column vector of observed signals. $A$ is a $M \times N$ mixed matrix required that $M \geq N$.

The goal is to extract independent source signals from mixed signals by finding separation matrix $W$ through some assumptions and constraints under the premise of unknown source signal $S(t)$ and mixed matrix $A$, which makes output $Y(t)$ an estimation to source signal $S(t)$. That is to say,

$$
Y(t)=W X(t)=W A S(t) \approx S(t),
$$

where $Y(t)=\left[y_{1}(t), y_{2}(t), \ldots, y_{N}(t)\right]^{T}$ is the estimation of source signals.

Generally, the normal ECG and VLP signal can be thought of as statistical independence with each other; thus VLP signal will be extracted through FastICA algorithm [11].

The basic model is shown in Figure 1.

\section{Methodology}

3.1. R-FastICA Algorithm. Traditional FastICA method is to estimate source signals based on negative entropy.

In order to improve separation precisely, negative entropy combined with related function as objective function is proposed in this paper. The updating formula of R-FastICA algorithm is vector gradient derived by the negative entropy combined with related function. The basic idea of R-FastICA algorithm requires that extracted signals are not only independent but also have high precision. Related function $\delta$ is introduced and defined by following formula:

$$
\delta=\lg \frac{\sum_{i=1}^{N} y_{i} \cdot y_{i}^{T}}{\operatorname{MSE}}
$$



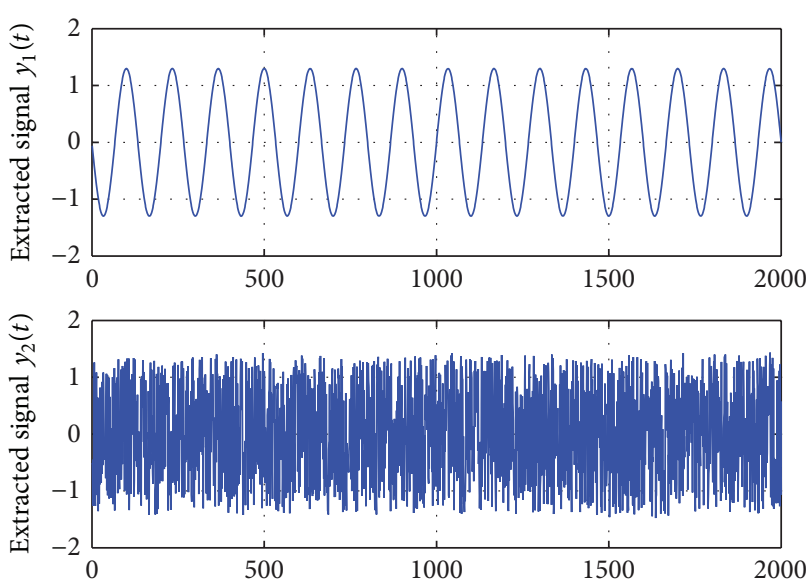

FIgure 4: Extracted signals with R-FastICA.
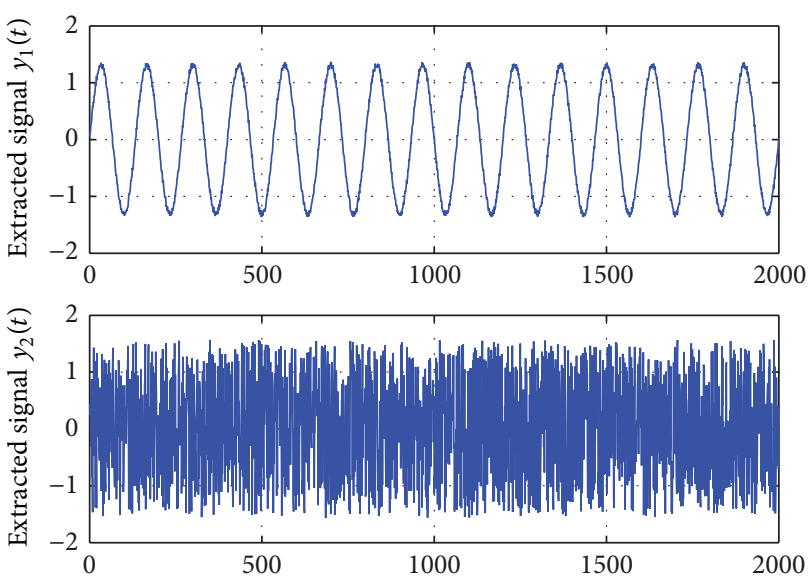

FIGURE 5: Extracted signals with traditional FastICA.

where MSE represents the mean square error between source signal and estimated signal, defined as [18]

$$
\text { MSE }=\sum_{i=1}^{N} \frac{\left(s_{i}-y_{i}\right)\left(s_{i}-y_{i}\right)^{T}}{N} .
$$

The MSE in type (3) is substituted by type (4), simplified as

$$
\delta=N \lg \frac{y \cdot y^{T}}{(s-y)(s-y)^{T}} .
$$

Source signal is replaced by the average of estimated signal. It is defined by $s=y^{*}=(1 / p) \sum_{j=1}^{p} y(t-j)$, where $p$ is an arbitrary integer less than 100 [19]. The type (5) can be simplified as

$$
\delta=N \lg \frac{y \cdot y^{T}}{\left(y^{*}-y\right)\left(y^{*}-y\right)^{T}} .
$$

BSS model is $Y(t)=W X(t)$ and $X^{*}$ is the average of $X$. It can be known that

$$
y^{*}=w^{T} X^{*}
$$

where $w$ is a column vector and $w^{T}$ is a row vector.

\begin{tabular}{|c|c|c|}
\hline & R-FastICA & FastICA \\
\hline \multirow{2}{*}{$\begin{array}{l}\text { Similarity } \\
\text { coefficient matrix }\end{array}$} & $1.0000 \quad 0.0002$ & $0.9995-0.0006$ \\
\hline & $0.0003 \quad 0.9999$ & $0.0004-0.9994$ \\
\hline
\end{tabular}

TABLE 1: The comparison of similarity coefficient matrix.

\begin{tabular}{lrllll}
\hline & \multicolumn{2}{c}{ R-FastICA } & \multicolumn{2}{c}{ FastICA } \\
\hline Similarity & -1.0000 & 0.0001 & 0.9996 & 0.0003 \\
coefficient matrix & 0.0003 & -1.0000 & 0.0005 & 0.9997 \\
\hline
\end{tabular}

TABLE 2: The comparison of similarity coefficient matrix.

Combining type (6) and type (7), it can be got that

$$
\begin{aligned}
\delta & =\lg \frac{w^{T} X \cdot\left(w^{T} X\right)^{T}}{\left(w^{T} X^{*}-w^{T} X\right)\left(w^{T} X^{*}-w^{T} X\right)^{T}} \\
& =\lg \frac{w^{T} X(X)^{T} w}{w^{T}\left(X^{*}-X\right)\left(X^{*}-X\right)^{\mathrm{T}} w} \\
& =\lg \frac{U}{V}=\lg U-\lg V,
\end{aligned}
$$

where

$$
\begin{aligned}
U & =w^{T} X(X)^{T} w \\
& =\left[w_{1} w_{2} \cdots w_{M}\right]\left[\begin{array}{c}
x_{1} \\
x_{2} \\
\vdots \\
x_{M}
\end{array}\right]\left[\begin{array}{llll}
x_{1} & x_{2} & \cdots & x_{M}
\end{array}\right]\left[\begin{array}{c}
w_{1} \\
w_{2} \\
\vdots \\
w_{M}
\end{array}\right] \\
& =w_{1}^{2} x_{1}^{T} x_{1}+w_{2} w_{1} x_{2}^{T} x_{1}+\cdots+w_{M}^{2} x_{M}^{T} x_{M} \\
& =\sum_{i=1}^{M} \sum_{j=1}^{M} w_{i} w_{j} x_{i}^{T} x_{j} .
\end{aligned}
$$

Similarly,

$$
\begin{aligned}
V & =w^{T}\left(X^{*}-X\right)\left(X^{*}-X\right)^{T} w \\
& =\sum_{i=1}^{M} \sum_{j=1}^{M} w_{i} w_{j}\left(x_{i}^{*}-x_{i}\right)^{T}\left(x_{j}^{*}-x_{j}\right) .
\end{aligned}
$$

From the above, $\delta$ is a function based on $w$ and $X$; the vector gradient can be obtained by the bottom of related function:

$$
\frac{\partial \mathrm{F}(w, X)}{\partial w}=\frac{\partial(\delta)}{\partial w}=\frac{1}{U} \frac{\partial U}{\partial w}-\frac{1}{V} \frac{\partial V}{\partial w}
$$

Vector gradient is defined as

$$
\frac{\partial g}{\partial w}=\left[\frac{\partial g}{\partial w_{1}}, \frac{\partial g}{\partial w_{2}}, \ldots, \frac{\partial g}{\partial w_{M}}\right]^{T}
$$



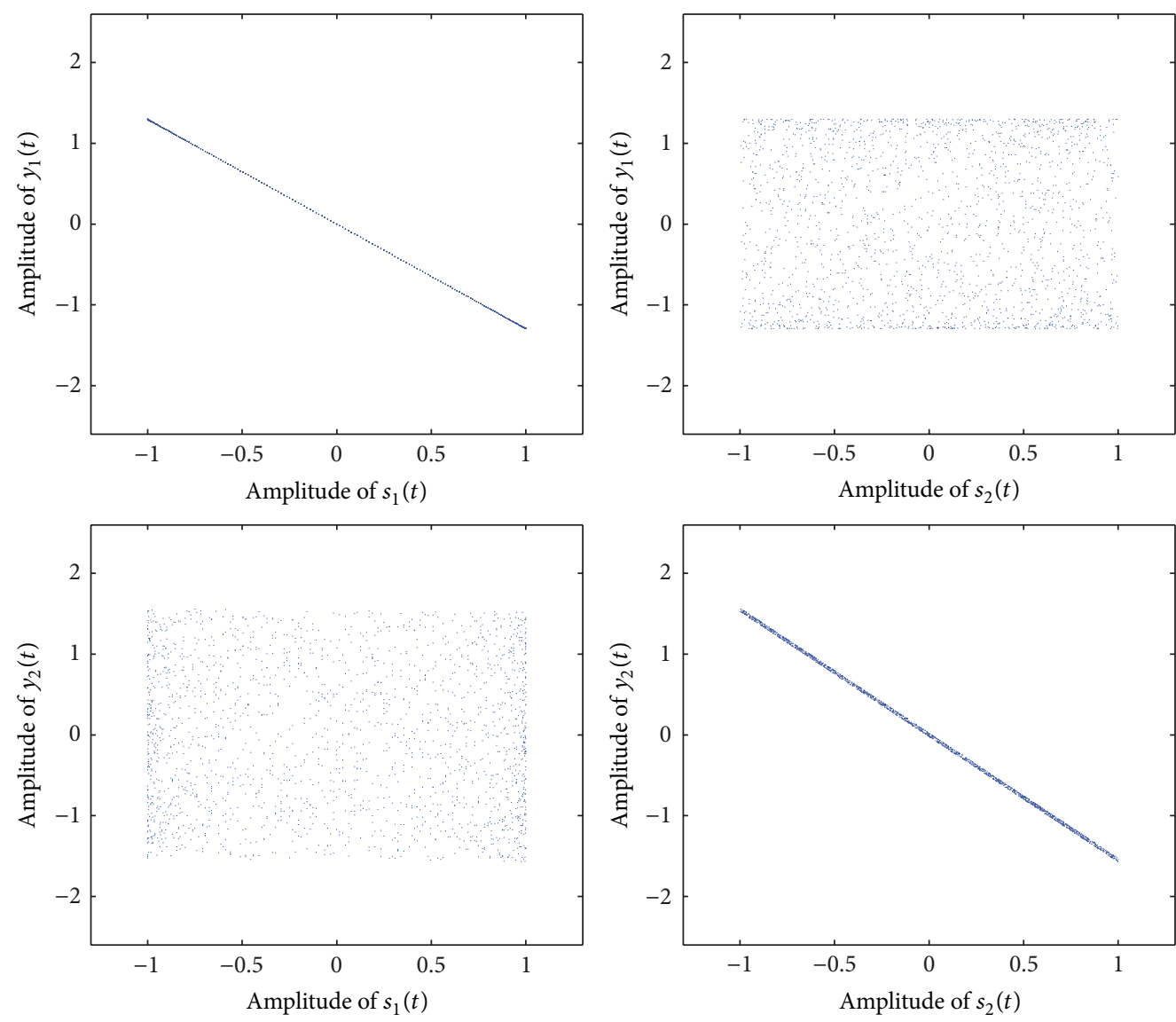

FIGURE 6: The composite scattering plot with R-FastICA algorithm.

The gradient of $U$ can be calculated according to type (12):

$$
\begin{aligned}
& \frac{\partial U}{\partial w}=\frac{\partial\left(\sum_{i=1}^{M} \sum_{j=1}^{M} w_{i} w_{j} x_{i}^{T} x_{j}\right)}{\partial w} \\
& =2\left[\sum_{j=1}^{M} w_{j} x_{1}^{T} x_{j}, \sum_{j=1}^{M} w_{j} x_{2}^{T} x_{j}, \ldots, \sum_{j=1}^{M} w_{j} x_{N}^{T} x_{j}\right] \\
& =\left[\begin{array}{ccccccc}
x_{1}^{T} & x_{1} & x_{1}^{T} & x_{2} & \cdots & x_{1}^{T} & x_{M} \\
x_{2}^{T} & x_{1} & x_{2}^{T} & x_{2} & \cdots & x_{1}^{T} & x_{M} \\
\vdots & & \vdots & & \ddots & & \vdots \\
x_{M}^{T} & x_{1} & x_{M}^{T} & x_{2} & \cdots & x_{M}^{T} & x_{M}
\end{array}\right]\left[\begin{array}{c}
w_{1} \\
w_{2} \\
\vdots \\
w_{M}
\end{array}\right] \\
& =2\left[\begin{array}{c}
x_{1}^{T} \\
x_{2}^{T} \\
\vdots \\
x_{M}^{T}
\end{array}\right]\left[\begin{array}{llll}
x_{1} & x_{2} & \cdots & x_{M}
\end{array}\right]\left[\begin{array}{c}
w_{1} \\
w_{2} \\
\vdots \\
w_{M}
\end{array}\right] \\
& =2 X X^{T} w \text {. }
\end{aligned}
$$

Similarly,

$$
\begin{aligned}
\frac{\partial \mathrm{V}}{\partial w} & =\frac{\partial w^{T}\left(X^{*}-X\right)\left(X^{*}-X\right)^{T} w}{\partial w} \\
& =2\left(X^{*}-X\right)\left(X^{*}-X\right)^{T} w
\end{aligned}
$$

Type (15) is a gradient of related function to $w$, which can be calculated combining type (13) with type (14):

$$
\frac{\partial F(w, X)}{\partial w}=2\left\{\frac{X X^{T} w}{w^{T} X X^{T}}-\frac{\left(X^{*}-X\right)\left(X^{*}-X\right)^{T} w}{w^{T}\left(X^{*}-X\right)\left(X^{*}-X\right)^{T} w}\right\}
$$

In literature [10], the approximate calculation formula of negative entropy is

$$
J(y) \propto\{E[G(y)]-E[G(v)]\}^{2} .
$$

The objective function is composed by negative entropy and related function including the information between source signal and estimated signal in R-FastICA:

$$
\varphi(y)=\{E[G(y)]-[G(v)]\}^{2} \cdot \delta,
$$



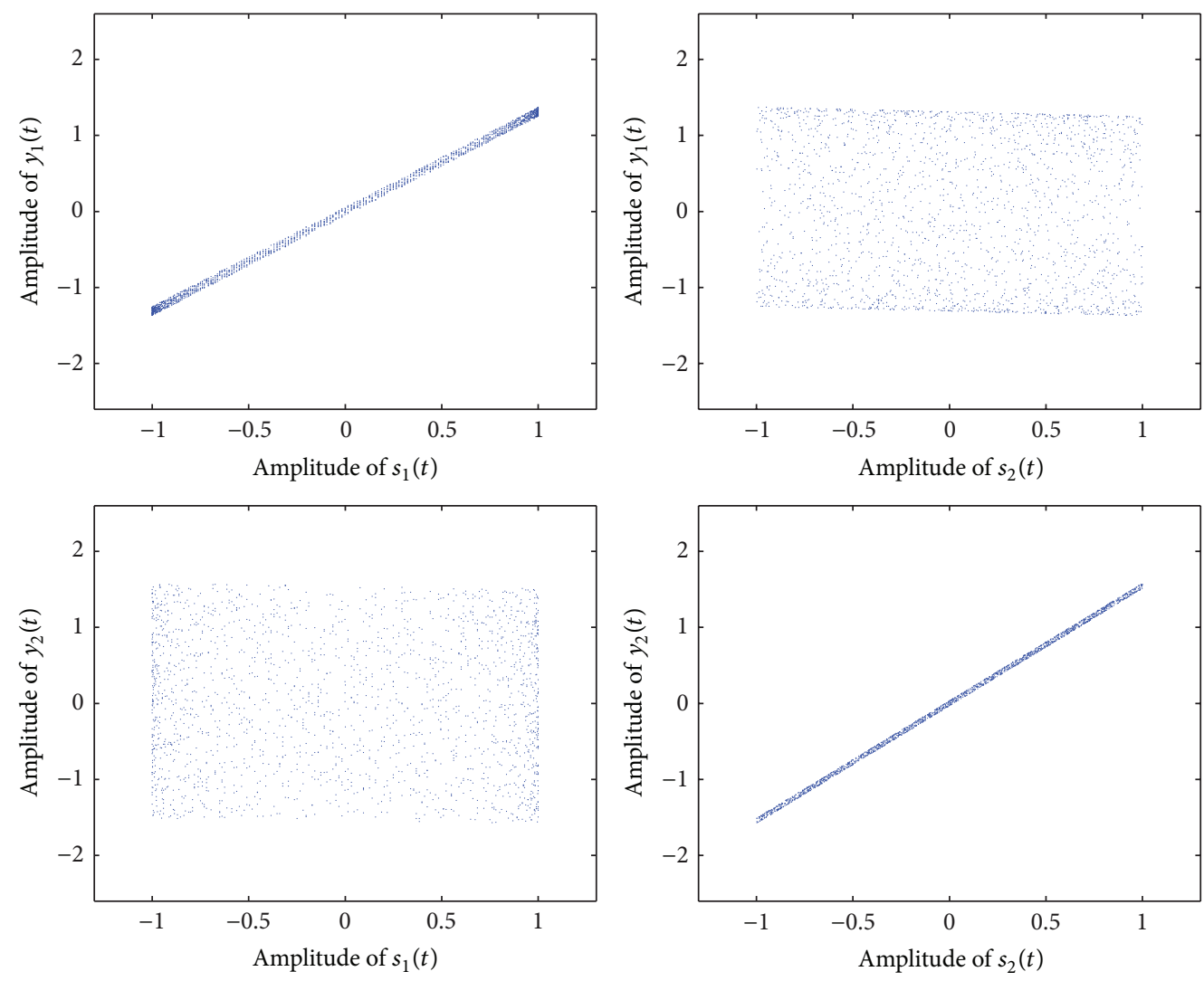

FIgURE 7: The composite scattering plot with FastICA algorithm.

where $v$ and $y$ are Gauss random variables with the same covariance (zero mean and unit variance) and $G$ is a nonlinear function selected by distribution form of source signals. follows:

The vector gradient of objective function $\varphi(y)$ to $w$ is as

$$
\begin{aligned}
\frac{\partial \varphi(y)}{\partial w}= & \frac{\partial\{E[G(y)]-E[G(v)]\}^{2} F(w, X)}{\partial w} \\
= & 2 \frac{\partial\{E[G(y)]-E[G(v)]\}}{\partial w} \\
& \cdot F(w, X)+\{E[G(y)]-E[G(v)]\}^{2} \cdot \frac{\partial F(w, X)}{\partial w} \\
= & 2\left\{E\left[X g\left(w^{T} X\right)\right]-E[G(v)]\right\} \\
& \cdot \lg \frac{w^{T} X X^{T} w}{w^{T}\left(X^{*}-X\right)\left(X^{*}-X\right)^{T} w} \\
& +2\{E[G(y)]-E[G(v)]\}^{2} \\
& \cdot E\left[\frac{X X^{T} w}{w^{T} X X^{T} w}-\frac{\left(X^{*}-X\right)\left(X^{*}-X\right)^{T} w}{w^{T}\left(X^{*}-X\right)\left(X^{*}-X\right)^{T} w}\right] .
\end{aligned}
$$

Type (18) is a new updating formula. The improved algorithm can ensure that the estimated signals are independent and the precision is higher due to the fact that related function $\delta$ is relevant to estimated signal.

3.2. Assessment Method. A familiar measure of separation performance is the similarity coefficient defined as [20]

$$
\varepsilon_{i j}=\varepsilon\left(y_{i}, s_{j}\right)=\frac{\left|\sum_{i, j=1}^{N} y_{i}(t) s_{j}(t)\right|}{\sqrt{\sum_{i=1}^{N} y_{i}^{2}(t) \sum_{j=1}^{N} s_{j}^{2}(t)}} .
$$

When $y_{i}=c s_{j}$, separation effect is ideal; when $y_{i}$ and $s_{j}$ are mutual independent, $\varepsilon_{i j}=0$; generally, similarity coefficient matrix is used to measure extracted performance.

The composite scattering plot [21] is a measure to describe corresponding relationship between the source signal and the extracted signal, where we can see that not only an extracted signal is the recovery of a source signal, but also the phase of source signal and extracted signal is the same or opposite.

\section{Simulation}

In order to indicate the performance of R-FastICA compared with traditional FastICA, the following simulations were conducted. 

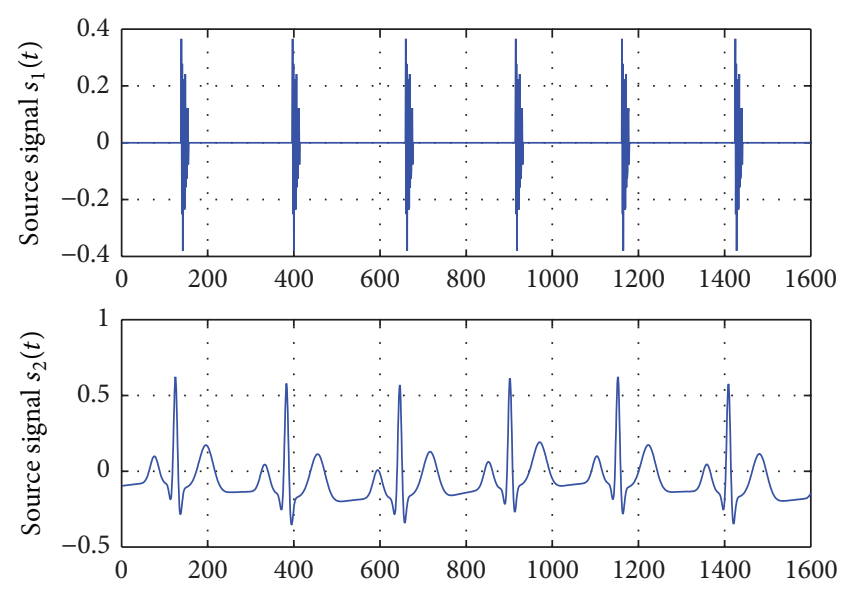

Figure 8: The source signals.
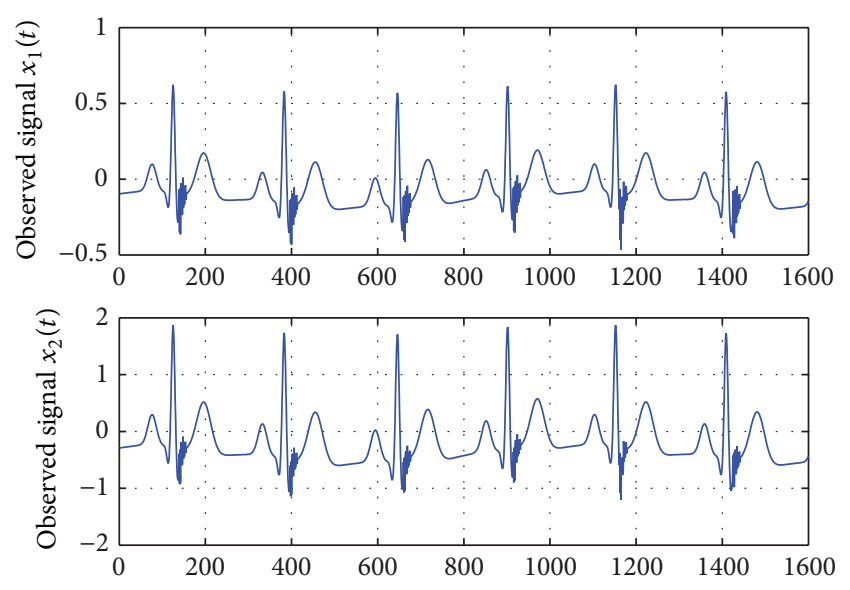

FIGURE 9: The observed signals.

Taking random signals as an example in the first simulation, R-FastICA method was proved to be effective. In the second simulation, taking ECG signal with VLP as an example, the original ECG signal without noises was from MIT/BIH database and VLP signal was generated through stacking sine waves with different frequency and amplitude [22].

4.1. Source Separation of the Random Signals. In this simulation, the source signal $s_{1}(t)$ was sinusoidal signal and $s_{2}(t)$ was random noises, whose sampling number was 2000. Signals $s_{1}(t)$ and $s_{2}(t)$ were shown in Figure 2, as well as mixed signals $x_{1}(t)$ and $x_{2}(t)$ that were shown in Figure 3.

The mixed signals were extracted through R-FastICA and traditional FastICA in Figures 4 and 5, respectively.

In the experiment of extracting random signals, the comparison of similarity coefficient matrix between RFastICA and FastICA algorithm was shown in Table 1.
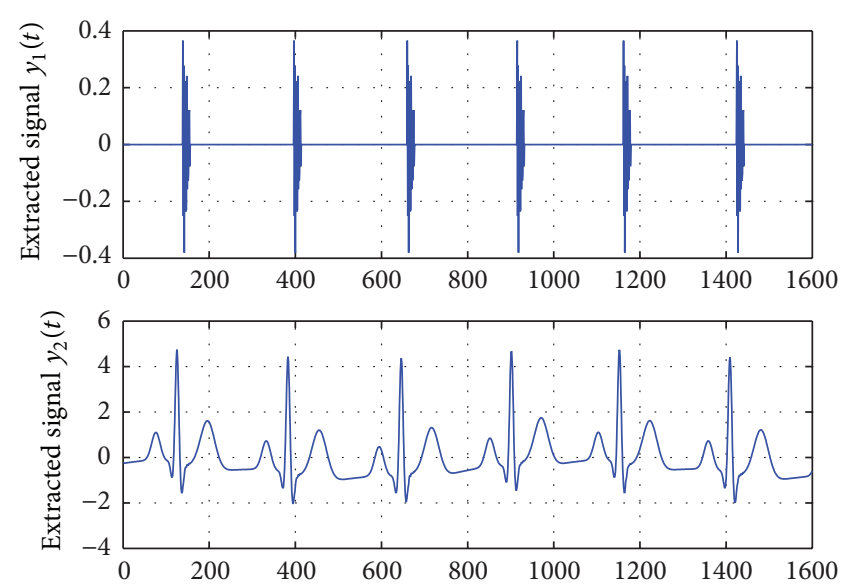

FIgURE 10: Extracted signals with R-FastICA.
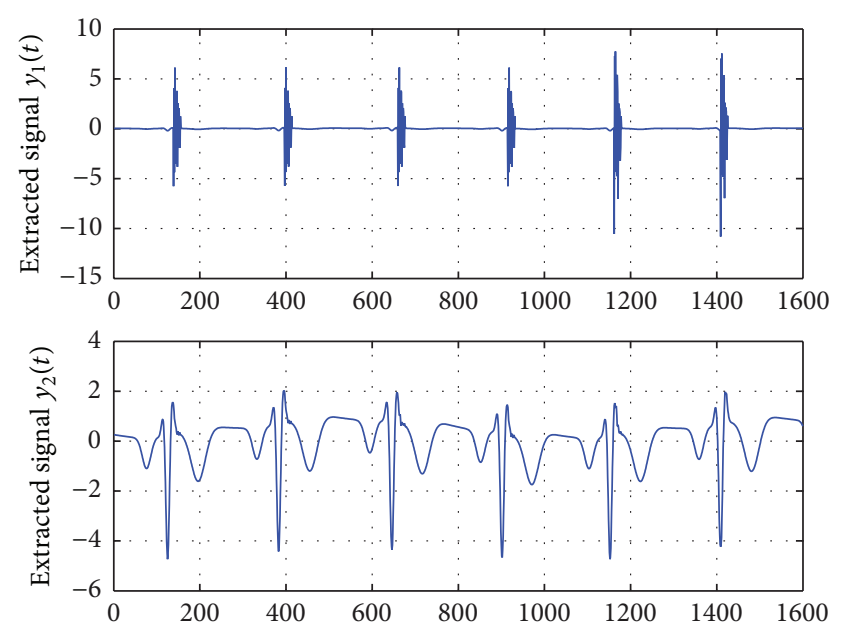

FIgURE 11: Extracted signals with FastICA.

Extracted signal $y_{1}(t)$ was the estimation of source signal $s_{1}(t)$ and the phase was opposite in Figure 6 and the same in Figure 7. Extracted signal $y_{2}(t)$ was the estimation of source signal $s_{2}(t)$ and the phase was opposite in Figure 6 and the same in Figure 7.

From the above experiments, we could see that R-FastICA algorithm outperforms traditional FastICA with higher similarity coefficient and separation precision.

4.2. Source Separation of the VLP Signal. In this simulation, the source signal $s_{1}(t)$ was VLP signal generated by program and $s_{2}(t)$ was ECG signal from MIT/BIH database, whose sampling number was 1600. The source signals $s_{1}(t)$ and $s_{2}(t)$, as well as mixed signals $x_{1}(t)$ and $x_{2}(t)$, were shown in Figures 8 and 9 , respectively.

The mixed signals were extracted through R-FastICA and traditional FastICA in Figures 10 and 11, respectively. 

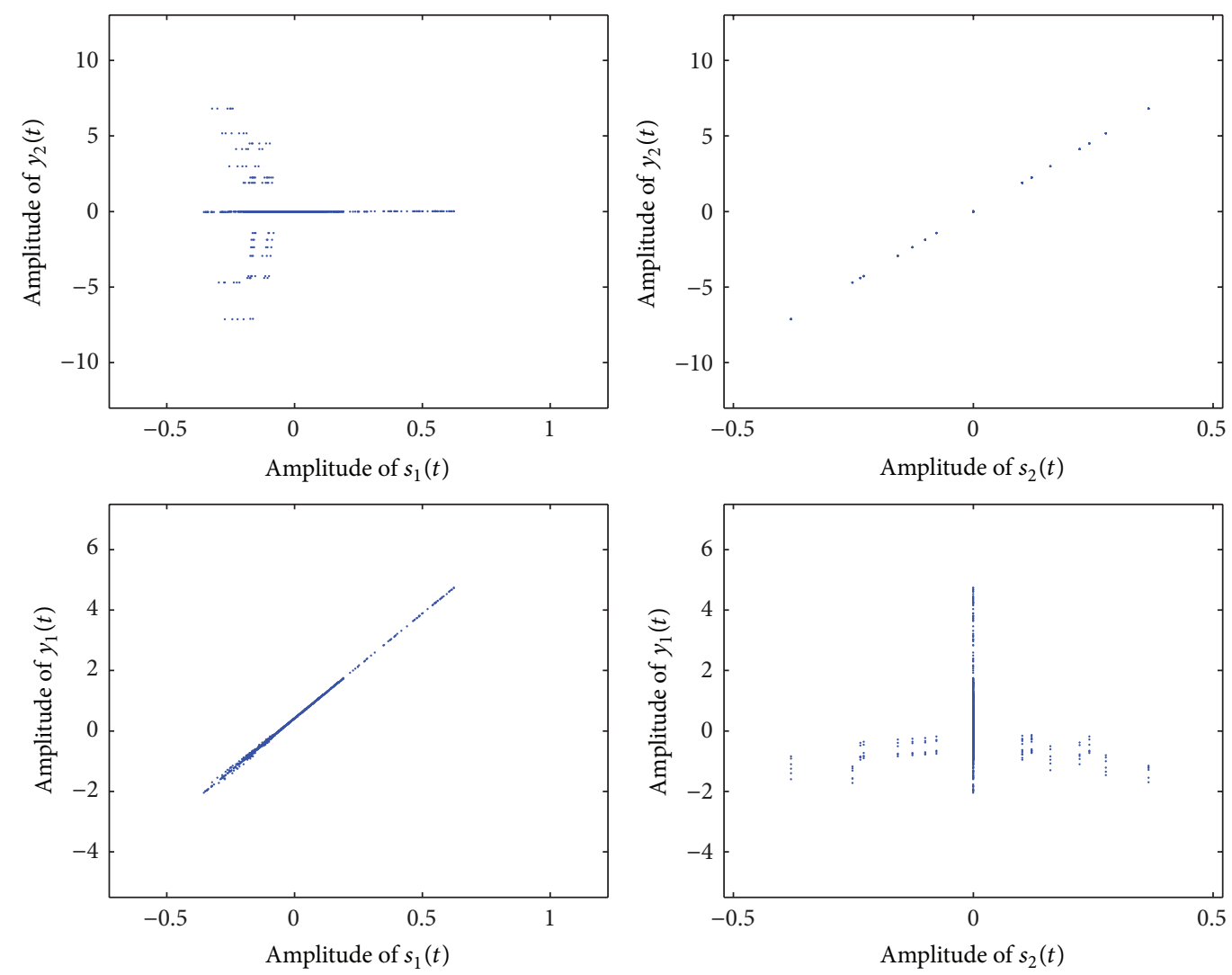

FIGURE 12: The composite scattering plot with R-FastICA.
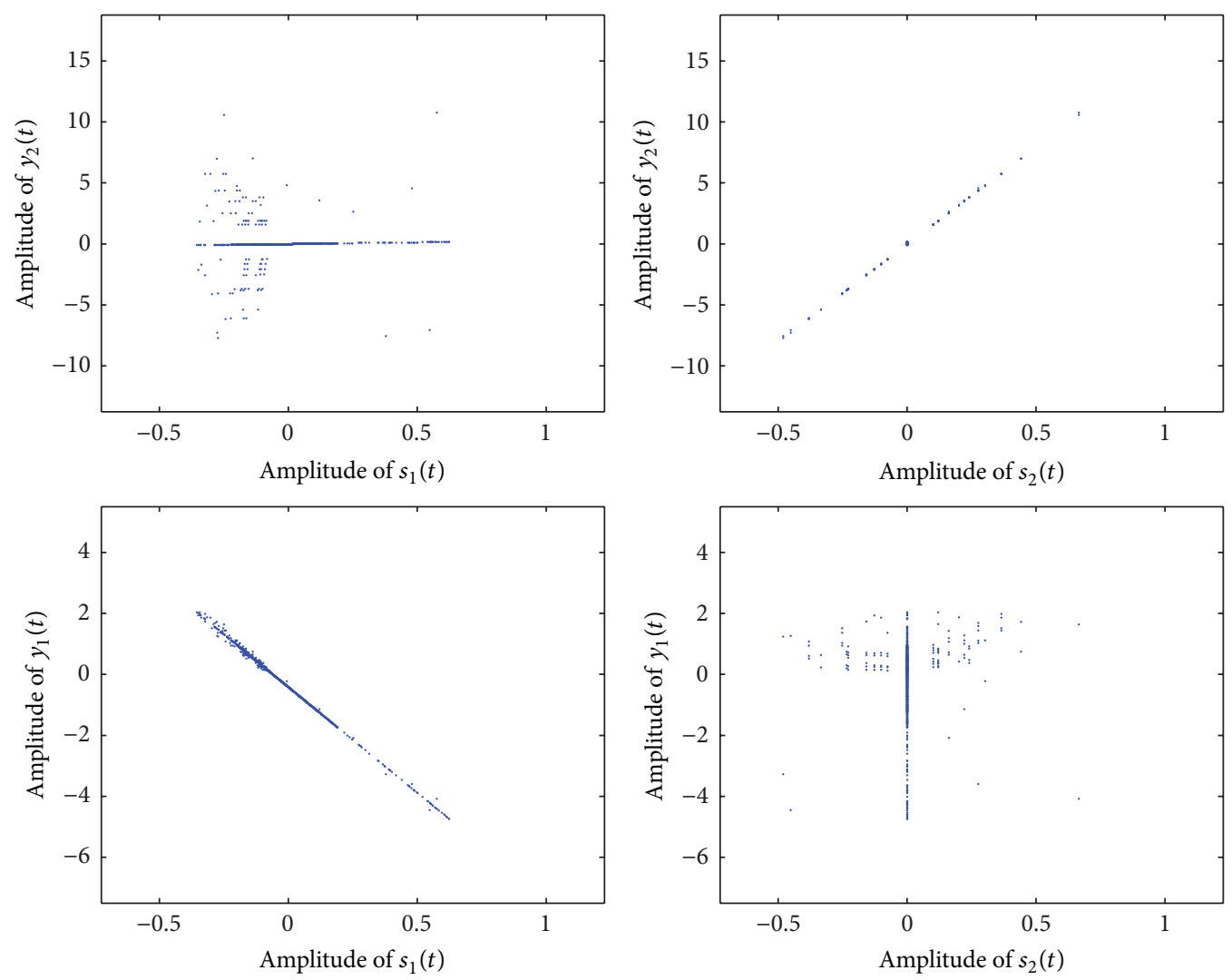

FIGURE 13: The composite scattering plot with FastICA. 
In the experiment of extracting VLP signal, the comparison of similarity coefficient matrix between RFastICA and FastICA algorithm was shown in Table 2.

Extracted signal $y_{1}(t)$ was the estimation of source signal $s_{1}(t)$ and the phase was the same in Figure 12 and the opposite in Figure 13. Extracted signal $y_{2}(t)$ was the estimation of source signal $s_{2}(t)$ and the phase was the same in Figures 12 and 13.

From the above experiments, the performance of RFastICA is superior to the FastICA obviously with higher similarity coefficient and high separation precision.

\section{Conclusion}

In this paper, R-FastICA algorithm and FastICA algorithm were adapted to extract random signals and to separate VLP signal from ECG signal. We believed that our study produced two important results. Firstly, we proposed a new method through the combination of related function and negative entropy and separated independent components by maximizing new objective function in the experiments. On the other hand, the experiments showed that R-FastICA method outperformed traditional FastICA method with higher similarity coefficient and high separation precision.

\section{Conflict of Interests}

The authors declare that there is no conflict of interests regarding the publication of this paper.

\section{Acknowledgments}

This project is supported by The General Object of National Natural Science Foundation (no. 61371062), Youth Science Foundation Project of National Natural Science Foundation (no. 61303207), Ministry of Education in 2012 Colleges and Universities by the Specialized Research Fund for the Doctoral Program of Joint Funding Subject (no. 20121402120020), Shanxi Province Science and Technology Development Project, Industrial Parts (no. 20120321024-01), Shanxi International Science and Technology Cooperation Project (no. 2012081031), Science and Technology Activities Project of Study Abroad Returnees in Shanxi Province in 2012 (Funded by Shanxi province human resources and social security hall), and Research Project supported by Shanxi scholarship council of China (no. 2013-032).

\section{References}

[1] C. J. James and C. W. Hesse, "Independent component analysis for biomedical signals," Physiological Measurement, vol. 26, no. 1, pp. R15-R39, 2005.

[2] Y. Feng, M.-Y. He, J.-H. Song, and J. Wei, "ICA-based dimensionality reduction and compression of hyperspectral images," Journal of Electronics \& Information Technology, vol. 29, no. 12, pp. 2871-2875, 2007.

[3] D.-W. Wang, H. Ji, and Y.-J. Wang, "Feature-level fusion recognition based on complex-valued independent component analysis," Optics and Precision Engineering, vol. 17, no. 8, pp. 2024-2031, 2009.

[4] X. Huang and H. Zeng, "Efficient variant of FastICA algorithm for speech features extraction," Lecture Notes in Electrical Engineering, vol. 113, pp. 929-935, 2012.

[5] H. Mehrabian, L. Lindvere, B. Stefanovic, and A. L. Martel, "A constrained independent component analysis technique for artery-vein separation of two-photon laser scanning microscopy images of the cerebral microvasculature," Medical Image Analysis, vol. 16, no. 1, pp. 239-251, 2012.

[6] A. J. Bell and T. J. Sejnowski, "An information-maximization approach to blind separation and blind deconvolution," Neural Computation, vol. 7, no. 6, pp. 1129-1159, 1995.

[7] X.-M. Yang, X.-D. Zheng, and X.-H. Liu, “The clinical significance of ventricular late potentials," Journal of Clinical Research, vol. 25, no. 11, pp. 1123-1124, 2012.

[8] A. S. Zandi and M. H. Moradi, "High-resolution ECG analysis: a fuzzy approach to detect ventricular late potentials using a wavelet-based vector magnitude waveform," in Proceedings of the IEEE Workshop on Signal Processing Systems-Design and Implementation, pp. 446-450, IEEE Press, November 2005.

[9] P. Bonato, R. Bettini, G. Speranza, F. Furlanello, and R. Antolini, "Improved late potential analysis in frequency," Medical Engineering and Physics, vol. 17, no. 3, pp. 232-238, 1995.

[10] F. Castells, J. Igual, J. J. Rieta, C. Sanchez, and J. Millet, "Atrial fibrillation analysis based on ICA including statistical and temporal source information," in Proceedings of the IEEE International Conference on Accoustics, Speech, and Signal Processing, pp. 93-96, IEEE Press, April 2003.

[11] H.-X. Zhang and L.-X. Jia, "The detection of ventricular late potential signal based on BSS," Journal of Jishou University (Natural Science Edition), vol. 29, no. 1, pp. 62-65, 2008.

[12] Y. Wang, The identification of ventricular late potentials based on wavelet transform and independent component analysis [M.S. thesis], Chongqing Univeersity, 2007.

[13] K. She, H.-M. Pu, F.-W. Zheng, and M.-T. Zhou, "Adaptive independent component analysis under multisensing," Journal of the University of Electronic Science and Technology of China, vol. 36, no. 1, pp. 11-13, 2007.

[14] Y. Song and D. Li, "Analysis method and search algorithm to ICA," Journal of System Simulation, vol. 21, no. 8, pp. 2346-2349, 2009.

[15] X.-X. Zhang and T.-S. Qiu, "Random variable analogy based ICA method," Journal of Electronics \& Information Technology, vol. 31, no. 9, pp. 2104-2108, 2009.

[16] F. R. Bach and M. I. Jordan, "Kernel independent component analysis," Journal of Machine Learning Research, vol. 3, no. 1, pp. $1-48,2002$.

[17] A. Hyvärinen and E. Oja, "Independent component analysis: algorithms and applications," Neural Networks, vol. 13, no. 4-5, pp. 411-430, 2000.

[18] Y.-Q. Du and H. Huang, "Fetal electrocardiogram extraction based on independent component and wavelet analyses," Journal of Clinical Rehabilitative Tissue Engineering Research, vol. 15, no. 13, pp. 2394-2397, 2011.

[19] J. Wan, X.-H. Zhang, J.-H. Rao, and Q.-P. Hu, "An independent component analysis algorithm using signal noise ratio," Control and Decision, vol. 27, no. 9, pp. 1376-1380, 2012.

[20] C. Vaya, J. J. Rieta, C. Sanchez, and D. Moratal, "Convolutive blind source separation algorithms applied to the electrocardiogram of atrial fibrillation: study of performance," IEEE 
Transactions on Biomedical Engineering, vol. 54, no. 8, pp. 15301533, 2007.

[21] Z.-H. Zhi, X.-Y. Zhang, and H.-R. Jia, "Flexible independent component analysis based on generalized gamma distribution," Journal of Taiyuan University of Technology, vol. 40, no. 1, pp. $4-7,2009$.

[22] R. Sivakumar, B. Muralikumar, B. Saranya, and V. P. Shanthiprabha, "Analysis of ventricular late potentials using wavelets and FFT spectrum," International Journal of Biomedical Engineering and Technology, vol. 6, no. 1, pp. 76-92, 2011. 


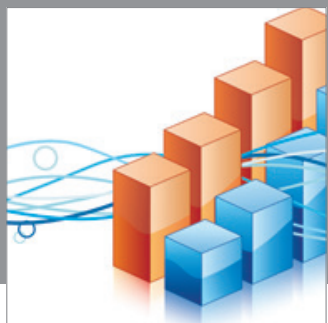

Advances in

Operations Research

mansans

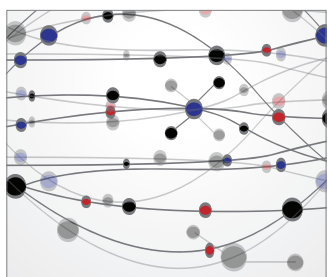

The Scientific World Journal
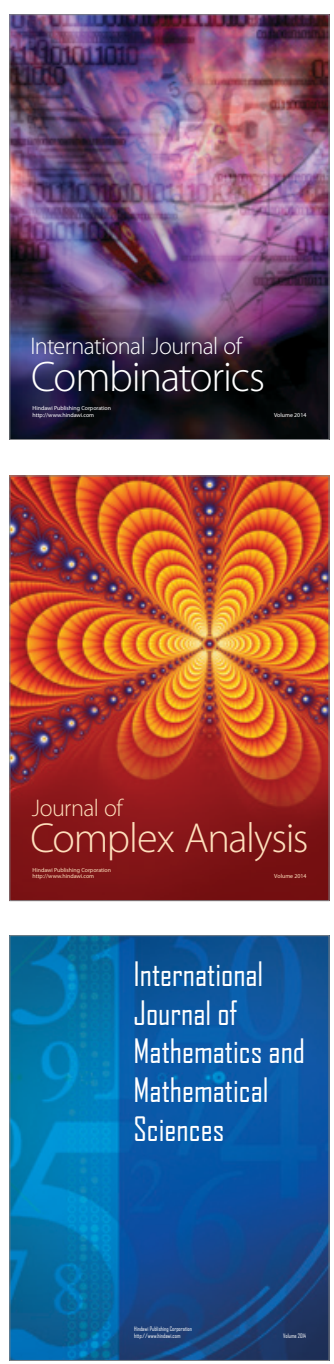
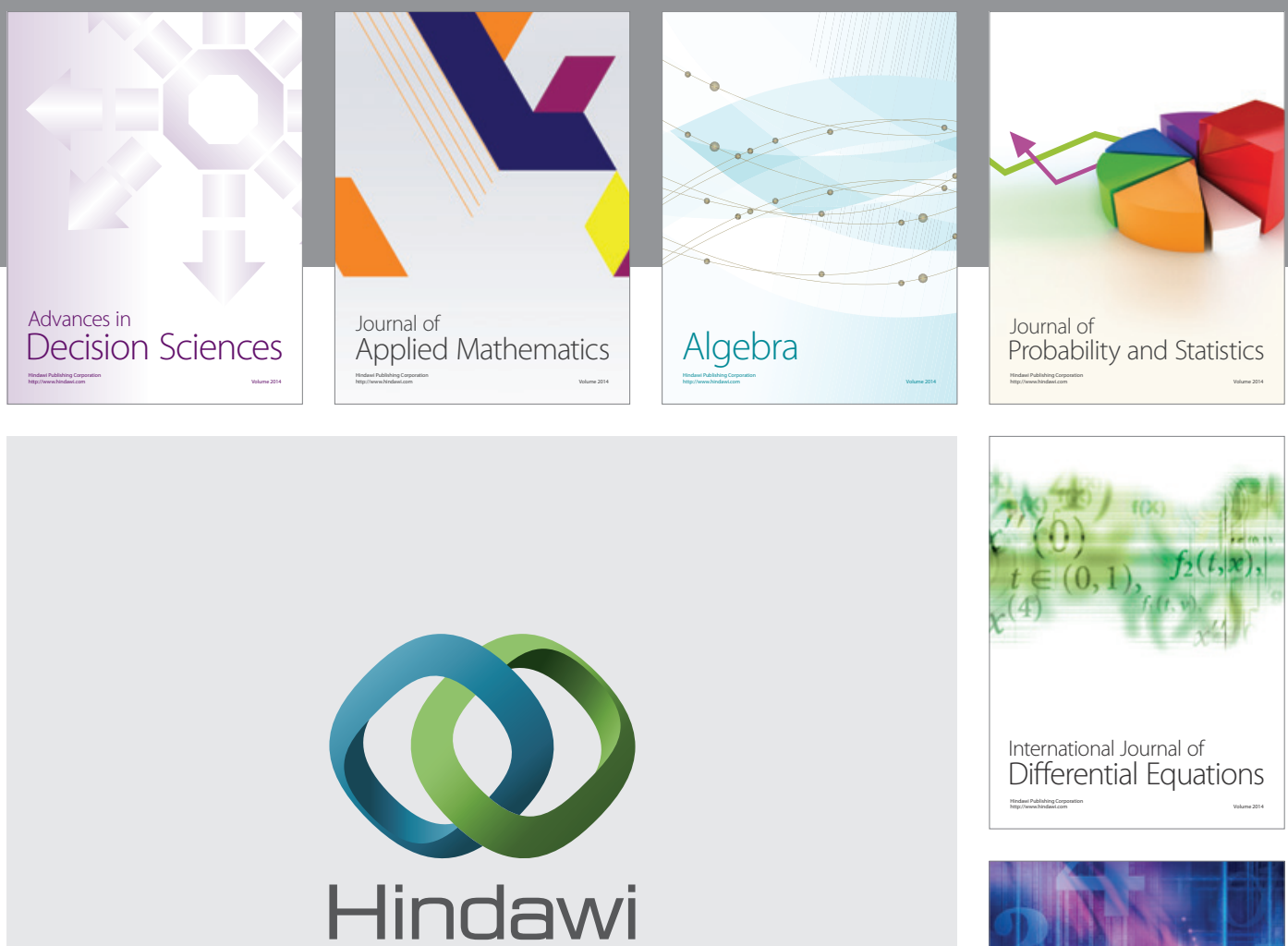

Submit your manuscripts at http://www.hindawi.com
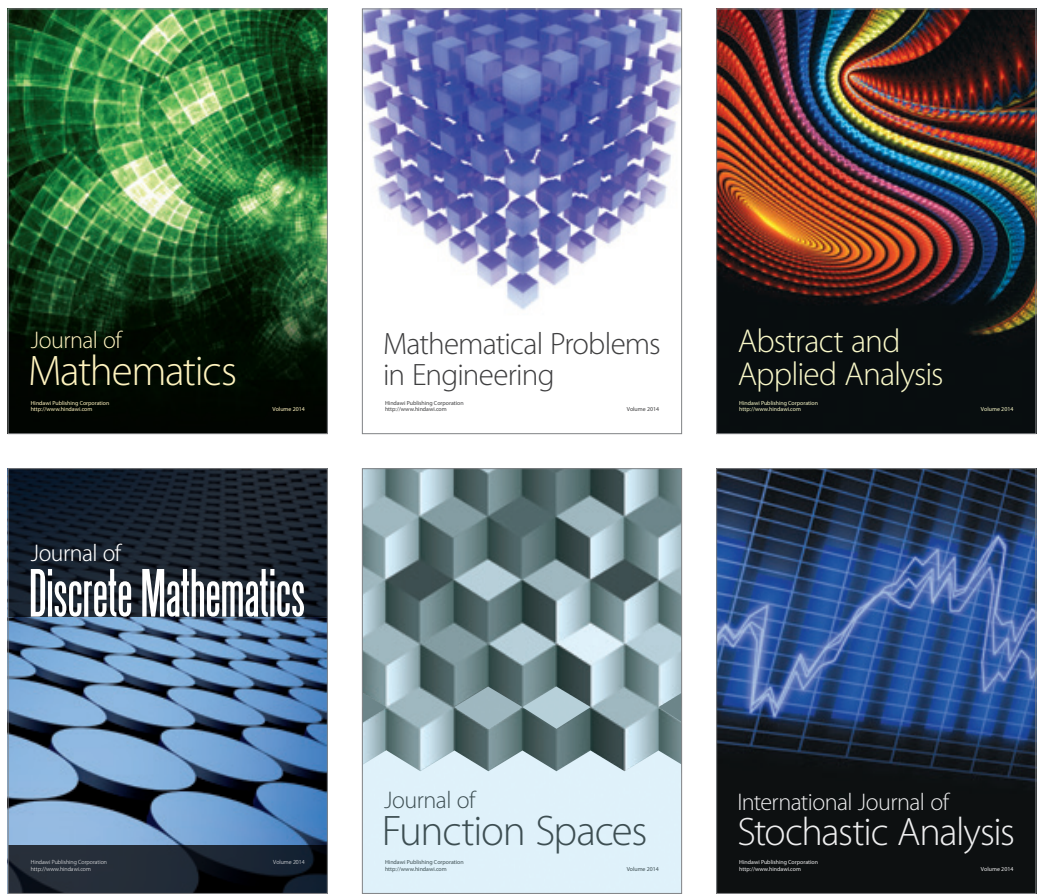

Journal of

Function Spaces

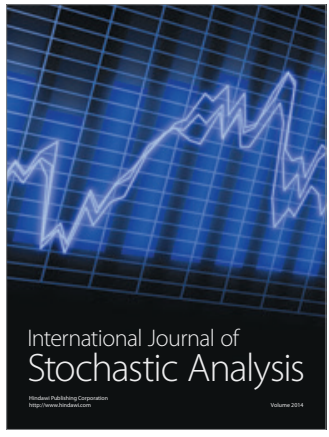

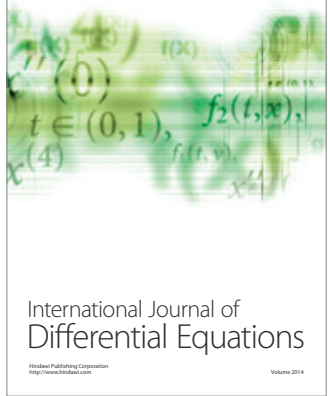
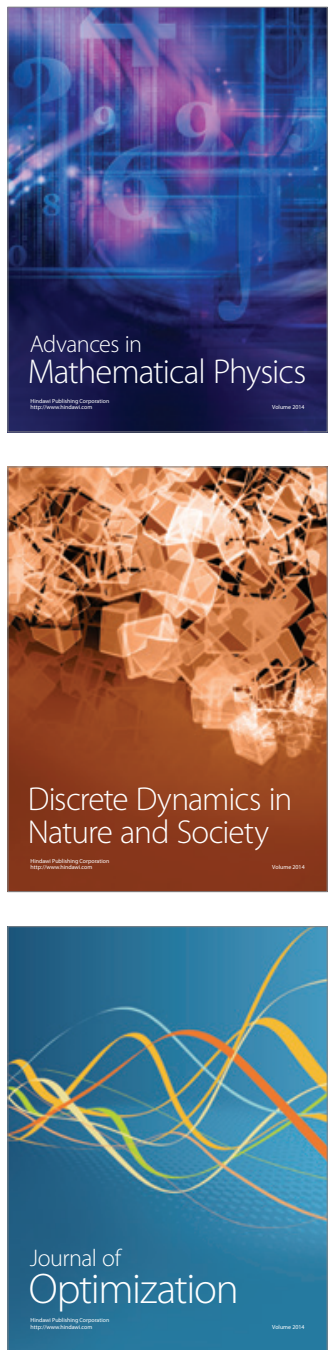